\title{
GENERAL DISCUSSION - FOURTH SESSION
}

Evans: It is essential to have a practical definition for a planetary nebula. Many brighter cases are disputed. The statistics of fainter objects (for which the possible observations are very limited) will be severely affected by the definition adopted. The practical definition now in use seems to be that a planetary nebula is an object which occurs in a catalogue of planetary nebulae.

Minkowski: In judging whether an object should be classed as a planetary nebula, the internal velocities should be taken into account. Unlike the planetary nebulae which show maximum velocities of expansion at their centres, NGC 6302 has zero velocity of expansion at the centre, and the North preceding loop seems to have been ejected with maximum velocity in the direction of its axis (Minkowski and Johnson, Astrophys. J., 148, 1967, 659).

As to the question of how to define a planetary nebula, there is no better way than to accept any object in a catalogue of planetary nebulae if nobody has serious objections.

Mathews: In view of the fact that stellar winds appear to be necessary in dynamical models of the nebula, it would seem that the appearance of NGC 6302 in the sky and its velocity field could both result from a stellar wind accelerating a model similar to that suggested by Khromov. If the initial ejection of nebular mass contained more mass in the equatorial region of a spherical shell, then the less massive polar regions would be more easily accelerated outward, expanding faster and reaching greater distances. Magnetic fields are therefore not needed to explain these apparent loops. Other nebulae such as NGC 6537, NGC 2346, NGC 650-1, and CD - 29 13998 are similar to NGC 6302 while nebulae like NGC 40 may represent a less advanced stage of this process. It would seem wrong, therefore, to suggest that NGC 6302 is uniquely unlike any other planetary and that it should be put into a class of its own.

Underhill: Arguments that planetary nebulae are the result of strong stellar winds are attractive. However, even if these winds do exist, they do not force us to conclude that all the central stars of planetary nebulae (odd-shaped gaseous bodies with nebular spectra) are at late evolutionary stages. Some Wolf-Rayet stars and some O stars are known to have stellar winds and to be surrounded by gaseous nebulae. These objects clearly cannot be placed at a very late stage of evolution. In other words, the characteristics of the surrounding nebula are not sufficient to determine the basic characteristic of the exciting star needed for stellar evolution studies.

Mathews: For mass-loss, the age of the star is not as important as the temperature and surface gravity. The observations of mass-loss by Morton refer, of course, to 
supergiants, but the same mechanism may produce mass-loss in planetary-nebula nuclei.

Abell: If we attempt to derive evolutionary tracks for central stars, and do so by comparing different nebulae, which are presumably in different stages of evolution, it is important to consider objects for which the stars, before ejection, all had nearly the same mass. This condition is satisfied by the majority of planetaries, which have a disk distribution, and thus probably have been ejected from stars of about 1.0 or 1.2 solar masses. But we also class as 'planetaries' objects surrounding, e.g., Wolf-Rayet stars, which quite probably have a history different from that of the more common disk-population planetaries. Ideally, I think a definition of a planetary nebula should include a specification of the kind of star that produced it.

Rose: Most planetary nebulae are observed to have low velocities of expansion $(10-100 \mathrm{~km} / \mathrm{sec})$. Perhaps the presence of low expansion velocities should be used in deciding if a peculiar object is a planetary nebula. In general, the expansion velocities for gas surrounding stars that are blue and not planetary nebulae (e.g. Wolf-Rayet stars) show considerably higher velocities of expansion.

Capriotti: Among the low surface-brightness planetary nebulae discovered by Abell, how many appear to be spherically symmetric?

Abell: About 56 out of 86 seem to be regular.

Capriotti: The implication has been made several times in this discussion that all planetaries evolve toward a more chaotic state, or at least toward a state of axial symmetry. However, from the Abell survey it appears that at least some of the planetary nebulae remain spherically symmetric, or evolve toward a state of spherical symmetry.

Abell: Most of the 86 planetaries that were found on the Palomar Sky Survey appear as fairly homogeneous disks or rings, but a large minority show irregularities, or are even highly chaotic. I agree with you that the morphological appearance should probably not be considered as fundamental in the definition of planetary nebulae, since it may merely represent different evolutionary stages, or perhaps only minor differences in the original density distributions within the nebulae. Far more fundamental is the kind (i.e. mass) of the star that produced a nebula, but of course this is not easy to know. Nevertheless, nebulae surrounding Wolf-Rayet stars should certainly not be included with other planetaries in studying the evolution of the involved stars.

Khromov: I would like to make a comment on the study of the structure of planetary nebulae. Due to stratification effects, the photographic images of a given planetary nebula can be very different in different spectral lines. If the images resulting from the light from several different ions in a planetary nebula are intermixed, due to the properties of the filter-plate combination that is used, the resulting structure of the nebula may appear highly complicated.

So, in studying the morphology, one should be sure to treat his observational material with a proper critical spirit. 
Menzel: I have the impression that every star might like to be a planetary nebula, but most of them simply do not make the grade. Our Sun, for example, is trying very hard, but it has succeeded in growing only feeble prominences, an inconspicuous corona, and an even less conspicuous solar wind. And yet, as I have pointed out elsewhere, our corona possesses many excitation features resembling those of planetaries: forbidden lines, filamentary structure, stratification, and a form suggestive of magnetic fields.

In my opinion only two main types of gaseous nebulae exist: diffuse and planetary. The former owe their luminosity to stars accidentally and temporarily wandering through them. The latter are closely associated generically with a central star. Such a star probably exists (or has existed) even when we cannot see it. The nebula owes its existence to the star. Therefore I think we should be generous in our definition of planetary nebulae. We should not tie it to some theoretical idea of the nature of the nebula or its nuclear star. If a star has an extended luminous envelope and if it appears in a catalogue of planetary nebulae, that is good enough for me.

Woltjer: I wish to comment briefly on what can be expected of magnetic fields in planetaries. For a field to be dynamically important it is first of all required that the magnetic energy be comparable to the kinetic and thermal energies. This implies a field of about $5 \times 10^{-4} G$. As to the nature of the field, it can be tied to the central star, it can have arisen from a stellar field by expansion, or it can have been produced by a field stretching mechanism, as in the solar wind. The latter can only give a field of small scale.

When we consider a field-containing region (with inner radius $R$ ) anchored in or close to the central star (mass $M$ ), we can show (Woltjer, L., Astrophys. J., 148, 1967, 291 ) that for a steady system we must have the magnetic energy $E_{\text {mag }}<\zeta G M^{2} / R$, with $\zeta$ a factor close to unity, provided the gravitational potential energy in the region outside $R$ is negligible and provided no pressure is applied from the outside. From this we find that a field that originates primarily in or close to the central star cannot have a strength of more than $3 \times 10^{-6} \mathrm{G}$ in a typical planetary shell. The same result can be demonstrated for a field resulting from the expansion of a stellar field. Our conclusion thus is that magnetic fields in planetaries, if present, are mostly of small scale. In this case they cannot significantly influence the large scale structure of the planetaries, which more probably is to be understood in hydrodynamic terms. Of course we do not exclude the idea that the ejection of matter from the star can be influenced by a stellar field.

In the outermost tenuous parts, the interstellar magnetic field can perhaps be of some importance. However, no large effects are expected because this field will not be compressed strongly around the planetary, since the expansion velocities are at most mildly supersonic with respect to the interstellar Alfvén velocity, in all but a few cases.

Finally we note that if a small scale field of $5 \times 10^{-4} G$ were present the diffusion of galactic high-energy electrons would lead to a non-thermal radio source of less than 
0.01 flux units at $400 \mathrm{Mc} / \mathrm{sec}$. Thus the eventual detection of non-thermal radio emission from planetaries would imply the local production of electrons with energy of several hundred $\mathrm{MeV}$.

Gurzadian: I wish to remind you that all observational data show that at the present time it is impossible to build a theory of the form of planetary nebulae without taking into account the role of magnetic fields, perhaps of the dipole type, connected with the shape of the nebulae. 